\title{
ON MODELLING OF ARTIFICIAL NETWORKS ARISING IN APPLICATIONS
}

\author{
Svetlana Atslega ${ }^{1,2}$, Felix Sadyrbaev ${ }^{2}$ \\ ${ }^{1}$ Latvia University of Life Sciences and Technologies, Latvia; \\ ${ }^{2}$ Institute of Mathematics and Computer Science, University of Latvia, Latvia \\ felix@latnet.lv
}

\begin{abstract}
We consider systems of two ordinary differential equations, which describe two-component Genomic Networks (GRN) and VNT (Virtual Network Topology) - controlled two dimensional telecommunication networks. VNT control over network is based on attractor selection like in biological (genomic) networks. We show that besides a set of stable critical points an attractor can be in the form of a stable periodic regime. We show the mechanism of emergence of a limit cycle through the Andronov-Hopf bifurcation. Similar attractors can appear in mathematical models with no limitations on dimensionality.
\end{abstract}

Keywords: nullclines, critical points, attracting sets.

\section{Introduction}

Core telecommunication networks nowadays are based on the Wavelength Division Multiplexing (WDM) technology. It allows transmitting multiple traffic channels over a single optic fiber. No additional equipment is required. Once carrying IP (the major Internet protocol) traffic over WDM network is to construct virtual network topology (VNT in short), which includes transmitting channels (lightpaths) and IP routers. The control over VNT requires a need to reconfigure VNT over a period of time in order to provide high-level service with minimal delays. Therefore VNT is to be adaptable to changes in traffic demand [1]. The majority of online VNT control methods are used when traffic demand is changing periodically and gradually [2]. This approach will not work, if changes of traffic demand are not predictable. There is a need for a VNT control method, which is adaptable to unknown changes in network environment. The new idea to invent the needed VNT control method was [3] to use the mechanism of attractor selection that is present in biological systems (organisms). This is based on attractor selection. Relatively general mathematical model for attraction selection is the system (1). This system in two-dimensional setting has appeared in studies of neuron networks. In ndimensional setting it describes interrelations in a genetic regulatory network(GRN) existing on a cell level in living organisms. The state of the biological network is described by the vector $x(t)$ and evolution of a network heavily depends on attractors of the system (1). Usually attractors are identified with stable equilibria (critical points) of the system (1). In our article we show that even in a twodimensional model (with only two equations in system (1)) attractors of more complicated nature can exist. We discuss also the mechanism of their emergence. This will allow for broader possibilities when arranging VNT control over telecommunication networks.

We consider systems of the form

$$
\left\{\begin{array}{l}
\frac{d x_{1}}{d t}=\frac{1}{1+e^{-\mu_{1}\left(w_{11} x_{1}+w_{12} x_{2}-\theta_{1}\right)}}-v_{1} x_{1} \\
\frac{d x_{2}}{d t}=\frac{1}{1+e^{-\mu_{2}\left(w_{21} x_{1}+w_{22} x_{2}-\theta_{2}\right)}}-v_{2} x_{2}
\end{array},\right.
$$

where $\mu_{1}>0, \mu_{2}>0, \theta_{1} \in R, \theta_{2} \in R, v_{1}>0, v_{2}>0$ - parameters

$w_{i j}$ - elements of the matrix

$$
W=\left(\begin{array}{ll}
w_{11} & w_{12} \\
w_{21} & w_{22}
\end{array}\right)
$$

This system has appeared in different contexts. In the work [3] (see also [4]) $x_{i}$ stands for populations of neurons that are decomposed in two groups, activating and inhibiting ones. In a series of papers [5-7] this system describes the two-component genomic regulatory network (GRN in short), where $W$ is a regulatory matrix. In this context system (1) contains information about dynamics of the elements of a network. The attracting sets are of particular interest. 
These are the main reasons for intensive studying systems of the form (1). Our purpose is to add some information about attractors in system (1) and similar systems of higher (than two) dimensions. For this, we consider system (1) with variable entries. We show how periodic solution (a limit cycle) can appear as the result of bifurcations of elements of the matrix $W$.

Our study has connections with papers [8-10], where similar problems were studied. The novelty of the current paper is showing how periodic solutions can appear and what the conditions for their existence are.

\section{Materials and methods}

It is convenient to treat system (1) on a phase plane. The nullcline method is used to gather the needed information. The nullclines are given by the equations

$$
\left\{\begin{array}{l}
x_{1}=\frac{1}{v_{1}} \cdot \frac{1}{1+e^{-\mu_{1}\left(w_{11} x_{1}+w_{12} x_{2}-\theta_{1}\right)}}-v_{1} x_{1} \\
x_{2}=\frac{1}{v_{2}} \cdot \frac{1}{1+e^{-\mu_{2}\left(w_{21} x_{1}+w_{22} x_{2}-\theta_{2}\right)}}-v_{2} x_{2}
\end{array} .\right.
$$

The function

$$
f(z)=\frac{1}{1+e^{-\mu z}}
$$

is sigmoidal. It is increasing, if $\mu$ is positive. It tends to zero or unity, as the argument $z$ goes respectively to $-\infty$ or to $+\infty$. There are papers, where different sigmoidal functions were used, for instance, the Gompertz function

$$
f(z)=e^{e^{-\mu z}}
$$

or the Hill's function

$$
f(z)=\frac{z^{\mu}}{z^{\mu}+\theta^{\mu}}
$$

It follows from (3) that the first nullcline is in the strip

$$
\left\{\left(x_{1}, x_{2}\right): 0<x_{1}<\frac{1}{v_{1}}, x_{2} \in R\right\}
$$

and the second one is in the strip

$$
\left\{\left(x_{1}, x_{2}\right): x_{1} \in R, 0<x_{2}<\frac{1}{v_{2}}\right\} .
$$

Therefore, all critical points are located in the open rectangle

$$
\left\{\left(x_{1}, x_{2}\right): 0<x_{1}<\frac{1}{v_{1}}, 0<x_{2}<\frac{1}{v_{2}}\right\} .
$$

there exists at least one critical point.

For analysis of critical points we use the linearized system. It is

$$
\left\{\begin{array}{c}
u_{1}^{\prime}=-v_{1} u_{1}+\mu_{1} w_{11} g_{1} u_{1}+\mu_{1} w_{12} g_{1} u_{2} \\
u_{2}^{\prime}=-v_{2} u_{2}+\mu_{2} w_{21} g_{2} u_{1}+\mu_{2} w_{22} g_{2} u_{2}
\end{array},\right.
$$

where

$$
g_{1}=\frac{e^{-\mu_{1}\left(w_{11} x_{1}^{*}+w_{12} x_{2}^{*}-\theta_{1}\right)}}{\left[1+e^{-\mu_{1}\left(w_{11} x_{1}^{*}+w_{12} x_{2}^{*}-\theta_{1}\right)}\right]^{2}}
$$




$$
g_{2}=\frac{e^{-\mu_{2}\left(w_{21} x_{1}^{*}+w_{22} x_{2}^{*}-\theta_{2}\right)}}{\left[1+e^{-\mu_{2}\left(w_{21} x_{1}^{*}+w_{22} x_{2}^{*}-\theta_{2}\right)}\right]^{2}}
$$

where $\left(x_{1}{ }^{*}, x_{2}{ }^{*}\right)$ - critical point under consideration.

Notice that $0<g_{i}<0.25$ for $i=1,2$. The coefficient matrix is

$$
A=\left(\begin{array}{cc}
\mu_{1} w_{11} g_{1}-v_{1} & \mu_{1} w_{12} g_{1} \\
\mu_{2} w_{21} g_{2} & \mu_{2} w_{22} g_{2}-v_{2}
\end{array}\right)
$$

and the characteristic equation is

$$
\begin{aligned}
& \operatorname{det}|A-\lambda I|=\left(\mu_{1} w_{11} g_{1}-v_{1}-\lambda\right)\left(\mu_{2} w_{22} g_{2}-v_{2}-\lambda\right)-\mu_{2} w_{21} g_{2} \mu_{1} w_{12} g_{1}= \\
& \mu_{1} \mu_{2} w_{11} w_{22} g_{1} g_{2}-\mu_{1} w_{11} g_{1} v_{2}-\mu_{1} w_{11} g_{1} \lambda-\mu_{2} w_{22} g_{2} v_{1}+v_{1} v_{2}+v_{1} \lambda-\mu_{2} w_{22} g_{2} \lambda+v_{2} \lambda+\lambda^{2}- \\
& \mu_{1} \mu_{2} w_{12} w_{21} g_{1} g_{2}=\lambda^{2}+\left(v_{1}+v_{2}-\mu_{1} w_{11} g_{1}-\mu_{2} w_{22} g_{2}\right) \lambda+\mu_{1} \mu_{2} w_{11} w_{22} g_{1} g_{2}- \\
& \mu_{1} w_{11} g_{1} v_{2}-\mu_{2} w_{22} g_{2} v_{1}-\mu_{1} \mu_{2} w_{12} w_{21} g_{1} g_{2}+v_{1} v_{2}=0 .
\end{aligned}
$$

To simplify, we can write the characteristic equation as

$$
\begin{gathered}
\lambda^{2}+B \lambda+C=0, \\
B=v_{1}+v_{2}-\mu_{1} w_{11} g_{1}-\mu_{2} w_{22} g_{2}, \\
C=\mu_{1} \mu_{2} w_{11} w_{22} g_{1} g_{2}-\mu_{1} w_{11} g_{1} v_{2}-\mu_{2} w_{22} g_{2} v_{1}-\mu_{1} \mu_{2} w_{12} w_{21} g_{1} g_{2}+v_{1} v_{2} .
\end{gathered}
$$

\section{Particular case}

We are interested in the particular case of $w_{12} w_{21}<0$.

Case 1. First set $w_{12}=w_{21}=0$. The regulatory matrix is

$$
W=\left(\begin{array}{cc}
0 & w_{12} \\
w_{21} & 0
\end{array}\right)
$$

and the system of differential equations takes the form

$$
\left\{\begin{array}{l}
x_{1}^{\prime}=\frac{1}{1+e^{-\mu_{1}\left(w_{11} x_{1}+w_{12} x_{2}-\theta_{1}\right)}}-v_{1} x_{1} \\
x_{2}^{\prime}=\frac{1}{1+e^{-\mu_{2}\left(w_{21} x_{1}+w_{22} x_{2}-\theta_{2}\right)}}-v_{2} x_{2}
\end{array} .\right.
$$

The characteristic equation is

$$
\begin{gathered}
\lambda^{2}+B \lambda+C=0, \\
B=v_{1}+v_{2} \\
C=-\mu_{1} \mu_{2} w_{12} w_{21} g_{1} g_{2}+v_{1} v_{2} .
\end{gathered}
$$

Notice that $C$ is positive due to our assumptions.

The roots of characteristic equation are

$$
\lambda_{1,2}=-\frac{B}{2} \pm \sqrt{\frac{B^{2}}{4}-C}>0
$$

The discriminant $D=B^{2} / 4-c$ is equal to

$$
\frac{1}{4}\left(v_{1}-v_{2}\right)^{2}+\mu_{1} \mu_{2} w_{12} w_{21} g_{1} g_{2} .
$$

Suppose $v_{1}=v_{2}$. Then $D$ is negative, $B$ is positive. Therefore the assertion. 
Proposition 1. If $v_{1}=v_{2}, w_{11}=w_{22}=0, w_{12} w_{21}<0$, a critical point is stable focus.

Remark 1. If the discriminant $D$ is positive, a critical point is a stable node.

Remark 2. If $w_{11}=w_{22}=0$, then the divergence of the vector field, defined by (1), is not zero and there are no closed orbits due to the Bendixsoncriterium [11].

Case 2. Set $w_{11}=w_{22}=k>0$ and still assume $w_{12} w_{21}<0, v_{1}=v_{2}>0$.

One has

$$
W=\left(\begin{array}{cc}
k & w_{12} \\
w_{21} & k
\end{array}\right)
$$

Proposition 2. If $v_{1}=v_{2}, w_{11}=w_{22}=k, w_{12} w_{21}<0$, a critical point is a stable focus for small positive $k$, the critical point is a center for some $\mathrm{k}=\mathrm{k}^{*}$, and the critical point becomes unstable focus for $\mathrm{k}>\mathrm{k}^{*}$ and close enough to $k$.

Proof by computing lambda-s for the new characteristic equation, taking into account that $g_{i}$ are separated from zero by a positive number.

The nullclines can intersect only once at a critical point $\left(x_{1}{ }^{*}, x_{2}{ }^{*}\right)$. Increase the parameter $k$. For $k=0$ the characteristic values $\lambda$ are complex conjugate with negative real parts $\operatorname{Re} \lambda$. There exists $k_{*}$ such that $\operatorname{Re} \lambda=0$ so the characteristic numbers are purely imaginary. If $k$ is increasing further, a unique critical point becomes unstable focus and a closed orbit (limit cycle) emerges. This is Andronov - Hopf bifurcation, where the bifurcation parameter is $k$. Suppose $k$ increases to the value $K$ such that the critical point remains unique.

Theorem. If $v_{1}=v_{2}, w_{11}=w_{22}=k$ increase, $w_{12} w_{21}<0$, a critical point suffers the Andronov Hopf bifurcation and a limit cycle emerges.

Proof by considering the Poincare section on one of the nullclines and showing that Poincare function changes sign. Therefore, a periodic solution exists. Two facts should be used: a) the unique critical point is unstable focus; b) the vector field on the boundary of the rectangle

$$
\left\{\left(x_{1}, x_{2}\right): 0<x_{1}<\frac{1}{v_{1}} .0<x_{2}<\frac{1}{v_{2}}\right\}
$$

is directed inward.

\section{Results and discussion}

We consider the system (1) with set of parameters: $\mu_{1}=10, \mu_{2}=5, w_{11}=k, w_{12}=-1, w_{21}=0.4$, $w_{22}=k, \theta_{1}=0.1, \theta_{2}=0.7, v_{1}=v_{2}=1$.

The system takes the form

$$
\left\{\begin{array}{c}
x_{1}^{\prime}=\frac{1}{1+e^{-10\left(k x_{1}-x_{2}-0.1\right)}-x_{1}} \\
x_{2}^{\prime}=\frac{1}{1+e^{-5\left(0.4 x_{1}+k x_{2}-0.7\right)}-x_{2}},
\end{array},\right.
$$

where $k \geq 0-$ a parameter.

All critical points located in the square $(0,1) \times(0,1)$.

If $k \in(0 ; 0.731092)$, the system has one critical point of the type stable focus.

For $k=0$ the system (20) has a critical point $(0.193733,0.0425932)$. The respective nullclines and the vector field can be found in Fig. 1. Blue line is the nullcline for $x_{1}$, that is, the curve, where $x_{2}^{\prime}=0$. Similarly, red line is the nullclinefor $x_{2}$, the curve where $x_{2}^{\prime}$ is zero. The same is meaning of blue/red lines in Fig. 3, Fig. 5 and Fig. 7. The characteristic values $\lambda=-1 \pm 0.356923 i$ are complex conjugate with negative real parts. The critical point is stable focus. The respective phase plane is shown in Fig. 2. 

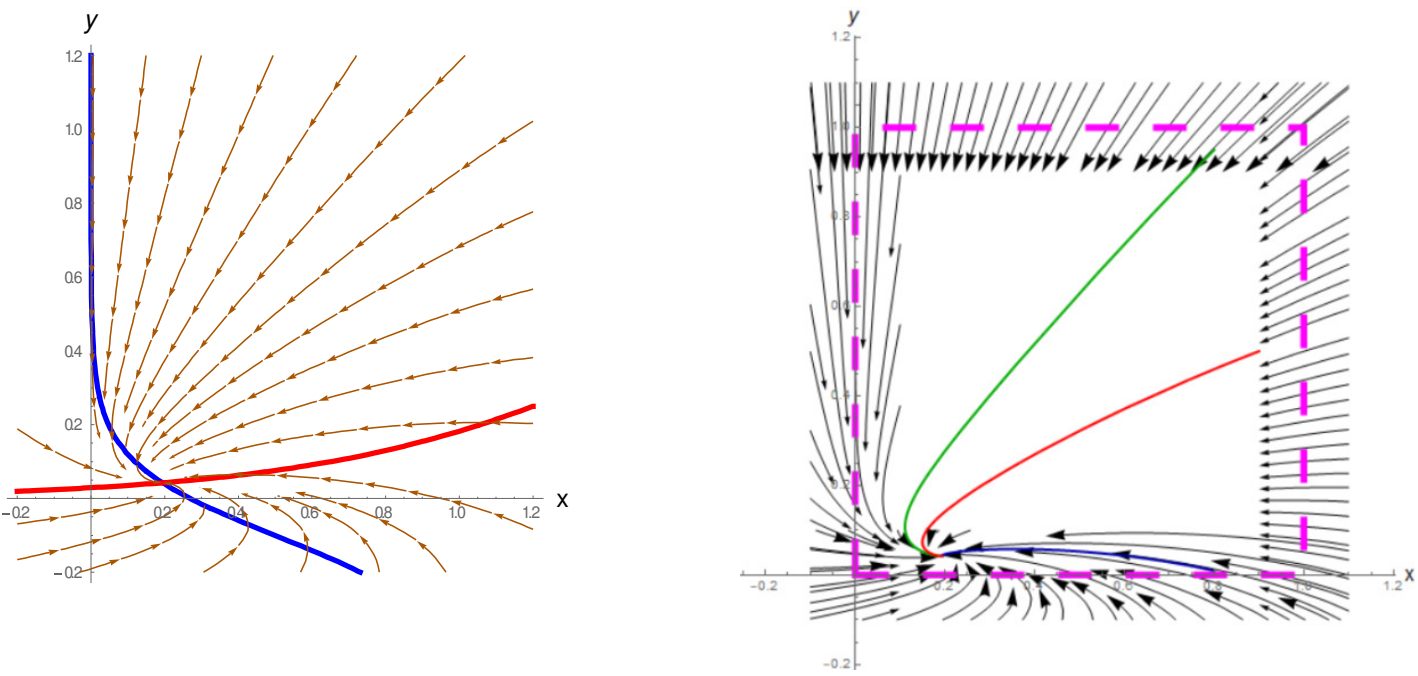

Fig. 1. Nullclines and the vector field for system (20), $k=0$

Fig. 2. Phase plane for system (20), $k=0$

If $k=0.731092$, the system has one critical point of the type center.

The system (20) has a critical point $(0.799337,0.346173)$. The respective nullclines and the vector field can be found in Fig. 3. The characteristic values $\lambda= \pm 0.834429 i$ are complex conjugate. The critical point is the center. The respective phase plane with centeris shown in Fig. 4 by red and blue colour.
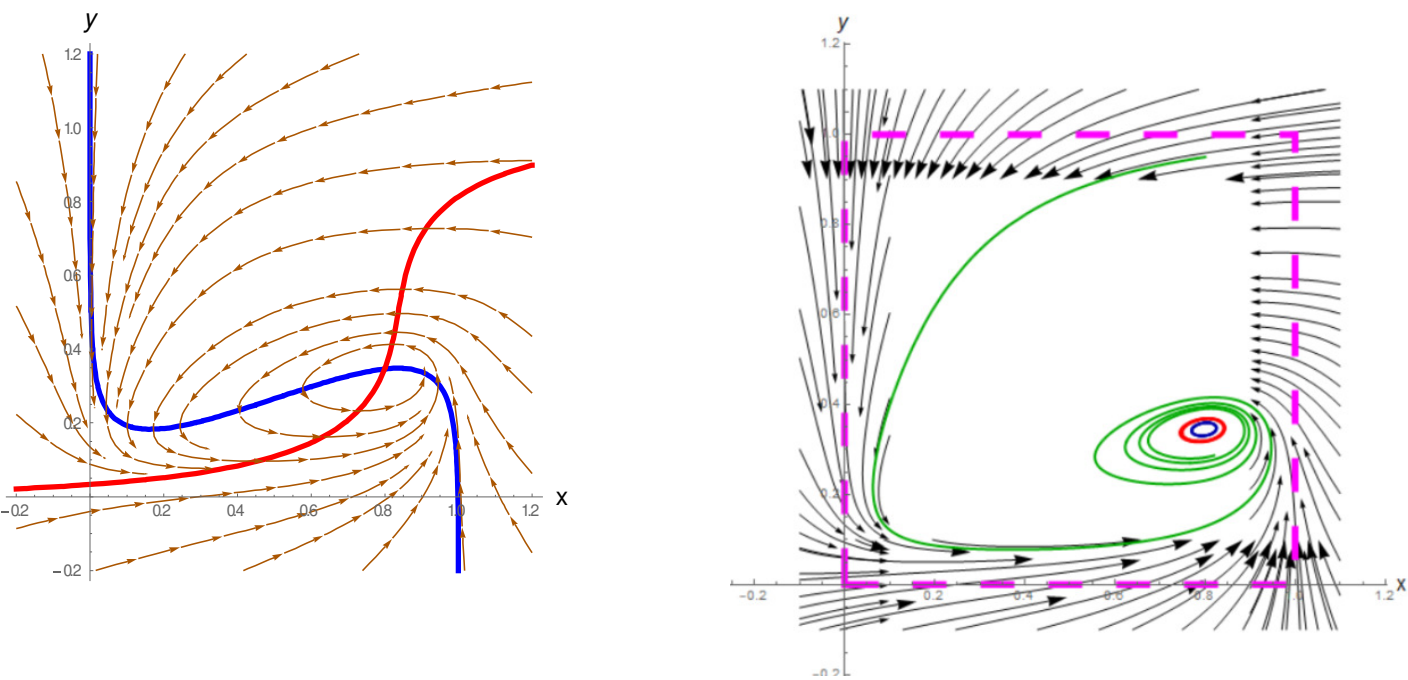

Fig. 3. Nullclines and the vector field for System (20), $k=0.731092$

Fig. 4. Phase plane for system (20), $k=0.731092$

If $k$ is increasing further, a unique critical point becomes unstable focus and limit cycle emerges.

For $k \in(0.731092 ; 1.21)$ the system has one critical point and limit cycle emerges.

If $k=0.8$ the system has the critical point $(0.746255,0.38913)$. The respective nullclines and the vector field can be found in Fig. 5. The characteristic values $\lambda=0.232849 \pm 0.905928 i$ are complex conjugate with positive real parts. The critical point is stable focus. The respective limit cycle (in red colour) along with the inner and outer trajectories (blue and green colours) is shown in Fig. 6. In practical situations of 2-element gene regulatory network the regulatory matrix $\left\{w_{11}=k, w_{12}=-1\right.$, $\left.w_{21}=0.4, w_{22}=k\right\}$ can be interpreted as follows: gene $x_{2}$ inhibits (suppresses) gene $x_{1}$ with intensity -1 , in turn gene $x_{1}$ activates gene $x_{2}$ with intensity $0.4, k$ is set to zero. If $k$ becomes large enough, the stable periodic regime is established. This means that expression of protein on both genes tends to be periodical. 


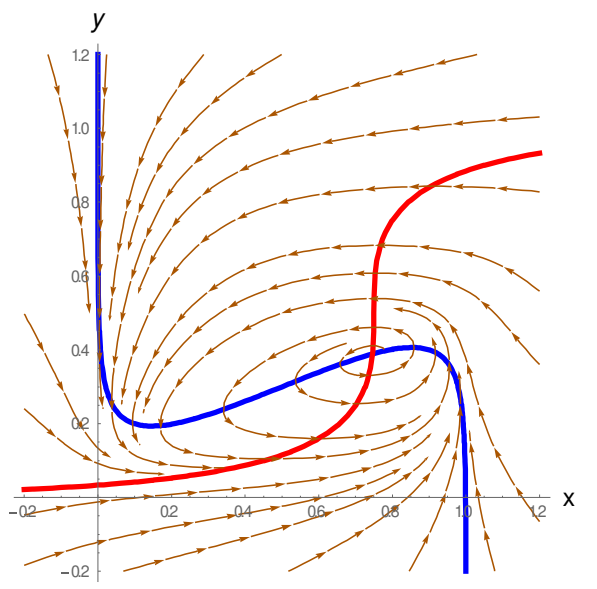

Fig. 5. Nullclines and the vector field for system (20), $k=0.8$

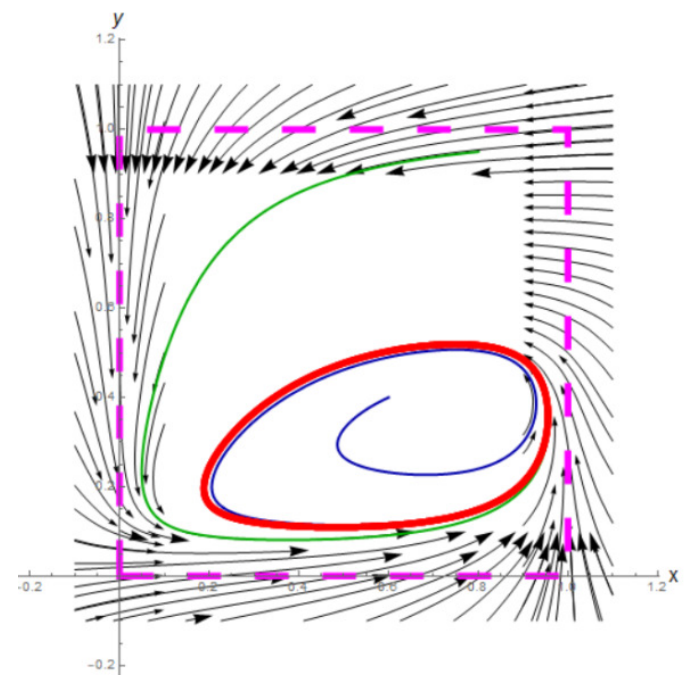

Fig. 6. Phase plane for system (20), $k=0.8$

If $k=1$, the system has the critical point $(0.537503,0.422473)$. The respective nullclines and the vector field can be found in Fig. 7. The characteristic values $\lambda=0.852941 \pm 0.901334 i$ are complex conjugate with positive real parts. The critical point is stable focus. The respective limit cycle (in red colour) along with the inner and outer trajectories (blue and green colours) is shown in Fig. 8.

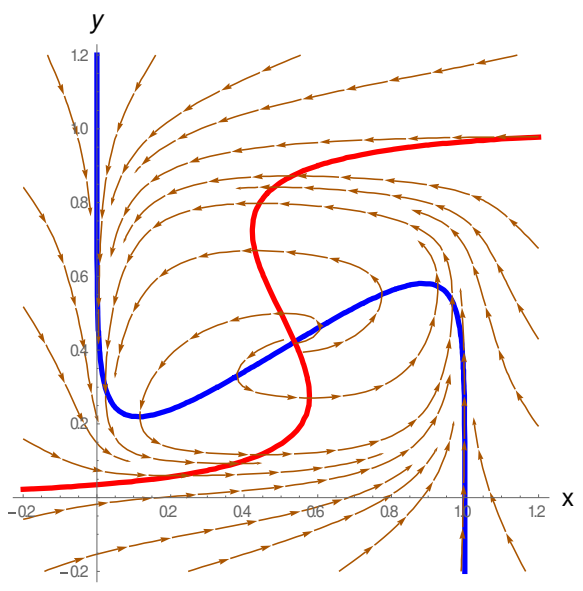

Fig. 7. Nullclines and the vector field for system (20), $k=1$

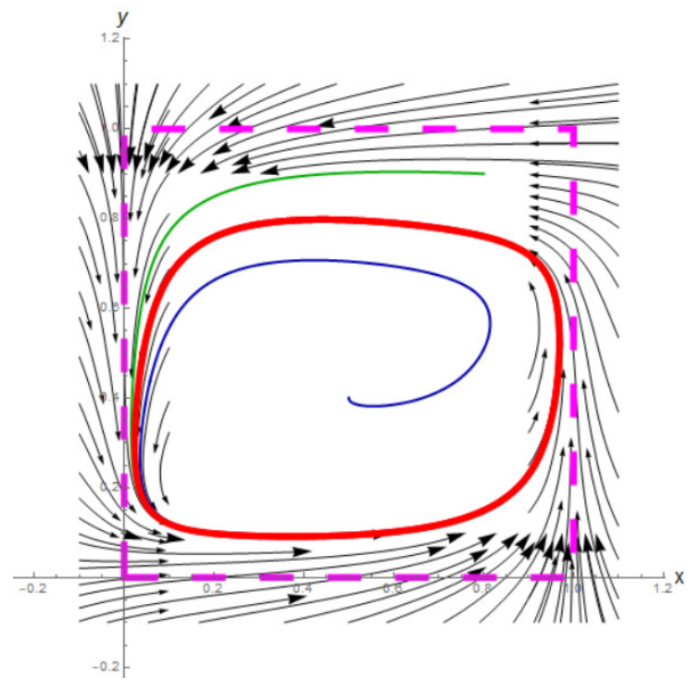

Fig. 8. Phase plane for system (20), $k=1$

\section{Conclusions}

The findings of the paper can be used when constructing VNT control over telecommunication networks that are subject to rapid and unpredictable changes. The system (1) is a two-component model of GRN. At the same time, it is a model of a two-component telecommunication network that consists of two pairs of nodes. The regulatory matrix (19) is interpreted as the Traffic demand matrix that shows how traffic flows are distributed via channels (lightpaths). The adaptivity properties of VNT control associated with system (1) (in a two-dimensional variant) rely on the structure and properties of attractors. Control built on a broader class of attractors will be more effective and rapid. It is possible to generalize results to high-dimensional systems and networks, considering systems with 2n-dimensional regulatory matrices, containing low-dimensional blocks of the form (19). 


\section{References}

[1] Finaskins D. Regulatory matrix influence on adaptability of attractor selection in virtual network topology control method. Proceedings The 55th International Scientific Conference of Daugavpils University, April 2013.

[2] Lakhina, A.Papagiannaki, K., Crovella, M., Diot, C., Kolaczyk, E.D. and Taft, N, June 2004. Structural analysis of network traffic flows. In Proceedings of ACM Sigmetrics, pp. 61-72.

[3] Wilson HR, Cowan JD. Excitatory and inhibitory interactions in localized populations of model neurons. Biophys J., vol 12 (1), 1972, pp. 1-24.

[4] Noonburg V.W. Differential Equations: From Calculus to Dynamical Systems, Providence, Rhode Island: MAA Press, 2019, $2^{\text {nd }}$ edition.

[5] Alakwaa F.M. Modeling of Gene Regulatory Networks: A Literature Review. J. Computational Systems Biology, vol. 1 (1), 2014, pp. 1- 8

[6] Jong H.D. Modeling and Simulation of Genetic Regulatory Systems: A Literature Review. J. Computational Biology, vol 9 (1), 2002, pp. 67-103.

[7] Vijesh N., Chakrabarti S.K., Sreekumar J. Modeling of gee regulatory networks: A review, J. Biomedical Science and Engineering, vol. 6, 2013, pp. 223-231.

[8] Atslega S., Finaskins D., Sadyrbaev F. On a Planar Dynamical System Arising in the Network Control Theory. Math. Modelling and Analysis, vol. 21 (3), 2016, pp. 385-398.

[9] Brokan E., Sadyrbaev F. On a differential system arising in the network control theory. Nonlinear Analysis: Modelling and Control, vol. 21 (5), 2016, pp. 687-701.

[10] Brokan E., Sadyrbaev F. Attraction in $n$-dimensional differential systems from network regulation theory. Mathematical Methods in Applied Sciences, vol. 41 (17), 2018, pp. 7498-7509.

[11]Lefschetz S. Differential equations: geometric theory. Interscience publ., 1957. 